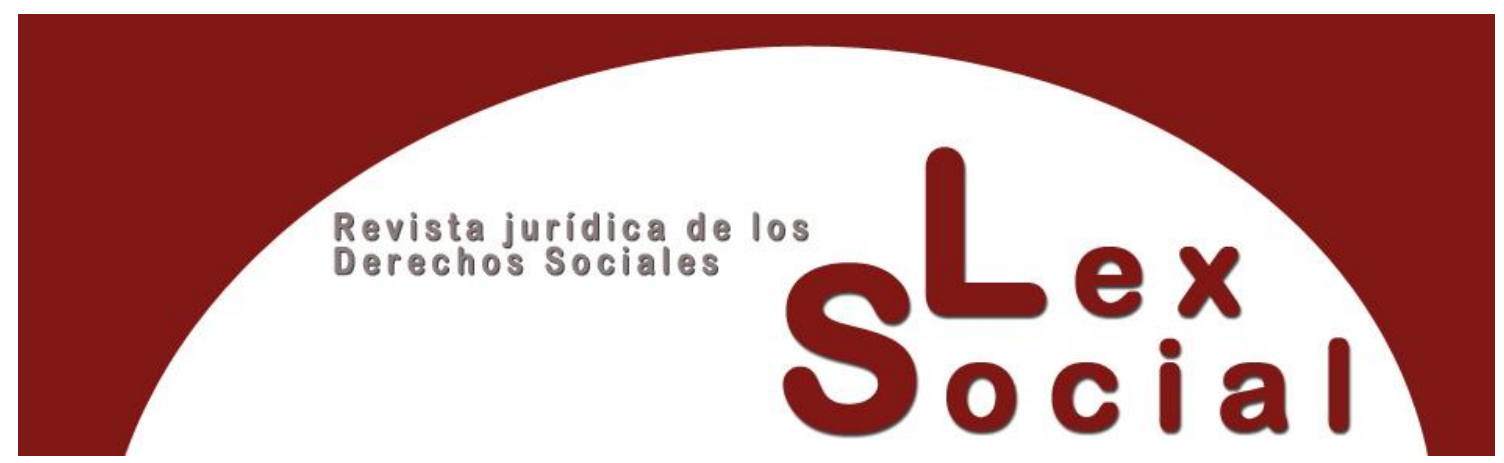

\title{
INSUFICIENCIA DEL MARCO DE PROTECCIÓN SOCIAL DE LOS CUIDADOS Y SUS EFECTOS EN LA SITUACIÓN LABORAL DE LAS MUJERES
}

\section{INSUFFICIENCY OF WORK-LIFE BALANCE POLICIES AND THE EFFECTS IN WOMEN CAREER}

\author{
PATRICIA NIETO ROJAS ${ }^{1}$ \\ Universidad Carlos III de Madrid \\ https://orcid.org/0000-0003-3734-3392
}

Como citar este trabajo: Nieto Rojas, P. (2021). Insuficiencia del marco de protección social de los cuidados y sus efectos en la situación laboral de las mujeres. Lex Social, Revista de Derechos Sociales, 11 (2), 804-823. https://doi.org/10.46661/lexsocial.5969

\section{RESUMEN}

Los cambios en el modelo de familia y la incorporación progresiva de casi tres millones de mujeres al mercado de trabajo hacen imprescindible una revisión del sistema tradicional de prestación de cuidados a aquellas personas que más lo necesitan, pues aunque el art. 39 CE obliga a la protección constitucional de familia, todavía hoy, el coste de oportunidad en el empleo de las personas con responsabilidades de cuidado se concentra en las mujeres, sin que la respuesta dada ni por el sistema de Seguridad Social ni por la Ley de Dependencia pueda calificarse como suficiente.

Palabras Clave: Seguridad Social; dependencia; menores de edad; conciliación; responsabilidad social.

\footnotetext{
${ }^{1}$ Este trabajo es el resultado del proyecto de investigación financiado por el Ministerio de Economía y Competitividad, "Cambio Tecnológico y Transformación en las Fuentes Laborales. Ley y Convenio colectivo ante la disrupción digital" dirigido por el profesor Mercader Uguina (RTI2018-094547-B-C21), y del proyecto dirigido por la profesora Molero Marañon, "Ser mujer en el mercado de trabajo", (RTI2018095398-B-I00).
}

\section{(c) ) EY-NC-SA}




\begin{abstract}
The changes in the family model and the progressive incorporation of almost three million women into the labor market are a challenge and introduce nowadays new factors in this situation. Although art. 49 of the Spanish Constitution promotes the constitutional protection of the family, however, the opportunity cost - in terms of access and maintenance in the employment of the persons with familiar responsibilities- is nowadays a verified effect; neither the answer given by the Social Security, nor the Dependence Act are enough.
\end{abstract}

KEY WORDS: Social Security; dependence; children; work-life balance; corporate social responsibility.

SUMARIO

I. Labores de cuidado y sus efectos en las trayectorias laborales de las mujeres

1. La insuficiencia de las prestaciones familiares como política de conciliación

2. La prestación para cuidado de hijos con cáncer o enfermedad grave

II. La tímida apuesta por la corresponsabilidad en las últimas reformas normativas

III. Las medidas para mitigar la brecha prestacional. El insuficiente alcance de las acciones impulsadas

IV. A modo de conclusión

Bibliografía

Anexo

"La figura que encarna al proletariado se ha transformado en la actualidad. Ya no es la del obrero en la cadena de montaje sino la de una mujer de piel oscura, una inmigrante que se ocupa de los niños y niñas, de las personas enfermas y de la gente mayor. "2

\title{
I. Labores de cuidado y sus efectos en las trayectorias laborales de las mujeres
}

El art. 39 CE declara la obligación de los poderes públicos de proteger a la familia e insta a que su desarrollo por el legislador ordinario garantice el fin último perseguido por la previsión constitucional ${ }^{3}$. La referida finalidad puede lograrse mediante ayudas directas de naturaleza económica, mediante reducciones fiscales o a través de la creación de

2 MOLINIER, P., “Género, trabajo y sexualidad”, en Laufer, L., Rochefort, F. (eds.) ¿Qué es el género?, Icaria, Barcelona, 2016, p. 152.

${ }^{3}$ Sobre este desarrollo legislativo, de obligada consulta CASTRO ARGÜELLES, M. A., "La protección familiar en el sistema español de Seguridad Social: un enfoque general”, en González Ortega, S. (ed.) Seguridad Social y Familia, La Ley, Madrid, 1999, pp. 92-104. 
servicios públicos de cuidado, siendo este tipo de acciones las que, a nuestro juicio, merecen una especial significación, pues son las más eficaces para permitir el mantenimiento en el mercado de trabajo de las personas que tengan a su cargo personas dependientes, pues los datos no solo evidencian la feminización de los cuidados, sino la expulsión de muchas mujeres del mercado de trabajo por la realización de esta función. Las personas que suministran cuidados sufren un enorme coste de oportunidad en términos laborales, ya que el tiempo que dedican a sus familiares no pueden destinarlo a otras actividades que podrían ser generadoras de ingresos (si se dedicara el tiempo a un trabajo remunerado o a trabajar a jornada completa en el caso de que trabaje a tiempo parcial), a conseguir mejores perspectivas de futuro (si empleasen el tiempo en reciclarse laboralmente o en actividades formativas) o, en general, a mejorar su calidad de vida $(\text { ocio, relaciones sociales...) })^{4}$.

La atención a las personas dependientes, aunque ciertamente no pueda explicar en toda su extensión la discriminación que sufren las mujeres en el mercado de trabajo ${ }^{5}$, es el factor que con más relevancia afecta no solo a su inserción sino a su mantenimiento en el mercado de trabajo en términos de igualdad, pues aun habiendo habido progresos en la corresponsabilidad masculina, el peso de la labor de crianza recae desproporcionadamente en las mujeres, y este es uno de los principales frenos para la consecución de una sociedad desde el punto de vista del género más equitativa.

Debido a sus menores salarios, "en el contexto de una desigual atribución de valor a los trabajos más feminizados y de un desigual reparto y asunción de tareas de cuidados por género y en el marco de un sistema de valores y actitudes que impulsa la asunción de estos roles por el género femenino, resulta más eficiente para las unidades decisorias, en general, las familias que, en caso de necesidad o dificultad para conciliar tiempos, sean las mujeres quienes salgan del mercado ${ }^{6 ",}$, máxime cuando las políticas de conciliación de la vida laboral y profesional, redactadas a partir de una titularidad neutra, han contribuido a perpetuar la tradicional asignación de roles, convirtiéndose, en la práctica, en medidas de sobrecarga para la mujer, por cuanto han provocado el consabido efecto de la doble jornada.

Urge, por tanto, introducir medidas que favorezcan la corresponsabilidad, lo que exige no solo que ambos sean titulares de los mismos derechos sino también obligaciones en relación a los cuidados de personas dependientes. Se ha constatado, a este respecto, que un factor muy relevante para fomentar la implicación de los hombres en el cuidado de los niños y en las tareas domésticas es la forma de disfrute del permiso, pues "cuando una

\footnotetext{
${ }^{4}$ Álvarez RamíreZ, G., Estudio sobre las necesidades de las familias con personas con discapacidad, Ministerio de Sanidad, Servicios Sociales e Igualdad, Madrid, 2015, p. 86.

${ }^{5}$ Ballester Pastor, M. A.; J. CABeza Pereiro, J., "Retos, expectativas y decepciones del derecho a la conciliación de responsabilidades", en Cruz Villalón, J. (ed.) Tutela y promoción de la plena integración de la mujer en el trabajo: libro homenaje a la profesora Teresa Pérez del Río, Consejo Andaluz de Relaciones Laborales, Sevilla, 2015, p. 107.

${ }^{6}$ Consejo Económico y Social. La participación laboral de las mujeres en España. Colección informes. Número 5/2016, p. 57.
} 
parte o todo se disfruta en solitario, esto es, asumiendo la plena responsabilidad del cuidado, una vez que la madre se reincorpora a su trabajo, su potencial para lograr estos objetivos aumenta sustancialmente"7. La Estrategia para la Igualdad de Género 20202025, de la Comisión Europea ${ }^{8}$, recuerda que "mejorar la conciliación de la vida privada y la vida profesional de los trabajadores es una de las formas de abordar las diferencias entre hombres y mujeres en el mercado de trabajo. Ambos progenitores tienen que sentirse responsables y facultados en lo relativo a las responsabilidades asistenciales".

La Directiva 2019/1158, relativa a la conciliación de la vida familiar y la vida profesional, pendiente de transposición por el ordenamiento jurídico español, también recuerda la relevancia del reparto equitativo de las responsabilidades asistenciales entre ambos progenitores e insta a los Estados miembros a que vayan más allá de estas normas mínimas en la revisión de sus políticas de conciliación de la vida familiar y laboral, toda vez que la incorporación masiva de las mujeres al mercado de trabajo ha puesto de manifiesto la necesidad de redistribuir las tareas y cuidados intrafamiliares entre los miembros de cada unidad familiar (corresponsabilidad) o "externalizarlos" y acudir a la prestación de servicios por personas ajenas a la familia, habida cuenta que "mientras unas mujeres se incorporan al mercado de trabajo, otras realizan el trabajo doméstico que aquellas no pueden afrontar en su integridad, de forma remunerada pero no siempre desde la economía productiva formal. No es de extrañar, por tanto, que solo gracias a la existencia de las trabajadoras domésticas es que muchas madres trabajadoras han podido incorporarse y/o progresar en la economía productiva formal; sin embargo, las primeras no siempre disfrutan de la protección necesaria para trabajar en condiciones laborales decentes como si lo hacen las segundas"9.

\section{La insuficiencia de las prestaciones familiares como política de conciliación}

Aunque las políticas de protección familiar han perseguido tradicionalmente tres objetivos: 1) la compensación económica por la asunción de cargas familiares; 2) el fomento de la natalidad y el equilibrio demográfico; y 3) la conciliación de la vida laboral y familiar, lo cierto es que las unidades familiares con hijos u otros familiares a cargo, especialmente si estos tienen una especial necesidad de cuidado, se encuentran en una situación desequilibrio entre ingresos y gastos derivada no tanto de una pérdida de ingresos (que como veremos, también) sino por el incremento de gastos que conllevan estas cargas familiares.

\footnotetext{
${ }^{7}$ Véase a este respecto los datos analizados en el Informe España 2020, p. 315, accesible desde: https://blogs.comillas.edu/informeespana/wp-content/uploads/sites/93/2020/10/Informe-Espana-2020Cap.-3-1.pdf

${ }^{8}$ COM (2020) 152 final. Comunicación de la Comisión al Parlamento Europeo, al Consejo, al Comité económico y social europeo y al comité de las regiones. Una Unión de la igualdad: Estrategia para la Igualdad de Género 2020-2025.

${ }^{9}$ GRAU PINEDA, C., La brecha de las pensiones en España, Bomarzo, Albacete, 2021, p. 91. 
La insuficiencia de servicios públicos de cuidado tiene un correlato directo en la forma en la que se produce la incorporación de la mujer al mercado laboral ${ }^{10}$ al hacer recaer sobre ellas los costes asociados a esta atención, y que se materializa en mayores tasas de inactividad, pero también en peores trayectorias profesionales ${ }^{11}$. Este hecho incrementa tanto la desigualdad de género como la desigualdad entre clases sociales, puesto que no todas las familias tienen la misma capacidad económica para acceder a estos servicios en el mercado privado ${ }^{12}$. Para mitigar este efecto, se ha instado al Gobierno a presentar un plan para la universalización de la escolarización desde el día siguiente a la finalización del permiso parental ${ }^{13}$, pero también a que impulse prestaciones económicas destinadas a compensar las peores trayectorias laborales de las mujeres que tienen responsabilidades de cuidado ${ }^{14}$.

Desde una perspectiva integral, cualquier política de protección a la familia tendría necesariamente que incluir también medidas que permitan a sus miembros, significadamente a las madres, que la función de cuidar y educar a los hijos (y en su caso, otros miembros de la unidad familiar dependientes) no se convierta en un obstáculo insalvable para poder desarrollar al mismo tiempo su vida personal y su carrera laboral y profesional $^{15}$. Sin embargo, en el modelo español, la insuficiencia de recursos públicos para el cuidado de personas dependientes, la escasa cuantía de la práctica totalidad de prestaciones familiares, hace que las mismas tengan "poco o nulo efecto sobre la conciliación de la vida familiar y laboral ${ }^{16 "}$. Y así podemos afirmar que aunque ciertamente las políticas familiares pretenden influir en los comportamientos demográficos, incrementando la tasa de natalidad mediante la eliminación de las dificultades, trabas o condicionantes (principalmente económicos) que están presentes en la función reproductora de las familias, parece evidente que esta finalidad no está siendo cumplida, salvedad hecha de la relevancia que tuvo para las familias con hijos enfermos la creación la prestación contributiva regulada para el cuidado de estos menores.

\footnotetext{
${ }^{10}$ RodRíGUez COPE, M. L., "Igualdad, conciliación, corresponsabilidad y flexibilidad", en Sáez Lara, C. (ed.) Igualdad de género en el trabajo: estrategias y propuestas, Laborum, Murcia, 2016, p. 70.

${ }^{11}$ Véase también CONSEJO ECONÓMICO Y SOCIAL, El cuidado de la primera infancia en los hogares españoles, p. 30.

${ }_{12}$ ÁlvareZ Ramírez, G., Estudio sobre las necesidades de las familias con personas con discapacidad, cit., p. 90.

${ }^{13}$ Proposición de Ley relativa a la reforma del sistema de permisos y prestaciones para el cuidado y atención de menores por parte de sus progenitores, en casos de nacimiento, adopción, guarda con fines de adopción o acogimiento (BOCG 17.1.2018).

${ }^{14}$ ILO, Care work and care jobs for the future of decent work, ILO, Geneva, 2018.

15 Almendros GonZÁlez, M.A., "La reforma de la coordinación de las prestaciones familiares de seguridad social como respuesta a los cambios políticos y económicos en la Unión Europea", Revista General de Derecho del Trabajo y de la Seguridad Social, vol. 49, 2018.

${ }^{16}$ MuÑoz MolinA, J., "La mujer en el ordenamiento de Seguridad Social", en Sáez Lara, C. (ed.) Igualdad de género en el trabajo: estrategias y propuestas, Laborum, Murcia, 2016, p. 192.
} 


\section{La prestación para cuidado de hijos con cáncer o enfermedad grave}

Hasta la reforma introducida por la DF $21^{\text {a }}$ de la Ley 39/2010, de Presupuestos Generales del Estado para el $2011^{17}$, el trabajador que necesitaba atender a un hijo afectado por otra enfermedad grave solo podía acudir a las siguientes vías:

a) la excedencia para atender al cuidado de un familiar hasta el segundo grado de consanguinidad o afinidad, que por razones de edad, accidente, enfermedad o discapacidad no pueda valerse por sí mismo, y no desempeñe actividad retribuida (art. $46.3 \mathrm{ET}$ ); o b) la reducción de jornada para encargarse del cuidado de un familiar en idénticas circunstancias.

Lo que ocurría es que ambas opciones presentaban inconvenientes importantes: la primera, por cuanto conlleva la pérdida temporal de la retribución (pues, al tratarse de una causa de suspensión del contrato, supone el cese de las dos obligaciones básicas de toda relación laboral: la obligación de trabajar y la de remunerar el trabajo) y presenta rigideces importantes con respecto a su duración (dado que no puede prolongarse por un período superior a dos años, aun cuando la necesidad de cuidados persista). La segunda, por cuanto la reducción de jornada conlleva una correlativa reducción del salario.

En la práctica, la atención de un menor afectado por cáncer u otra enfermedad grave venía conllevando, en demasiadas ocasiones, el abandono del mercado de trabajo de las madres de estos enfermos ${ }^{18}$; razón que hace sumamente plausible la creación de la prestación prevista en el RD 1148/2011, de 29 de julio, para la aplicación y desarrollo, en el sistema de la Seguridad Social, de la prestación económica por cuidado de menores afectados por cáncer u otra enfermedad grave ${ }^{19}$, que considera situación protegida la reducción de la jornada de trabajo que lleven a cabo las personas progenitoras, adoptantes y acogedoras de carácter familiar preadoptivo o permanente, cuando ambas trabajen, para el cuidado del menor a su cargo afectado por cáncer u otra enfermedad grave incluida en el listado que figura en el anexo del propio texto reglamentario.

Esta nueva prestación tiene por objeto compensar la pérdida de ingresos que sufren las personas interesadas al tener que reducir su jornada, con la consiguiente disminución del salario, por la necesidad de cuidar de manera directa, continua y permanente de los hijos o menores a su cargo.

Respecto a su presupuesto objetivo para el otorgamiento de esta prestación, con naturaleza de subsidio, se condiciona a que la enfermedad que padezca el menor implique un ingreso hospitalario de larga duración que requiera su cuidado directo, continuo y permanente, y que se considerará asimismo como ingreso hospitalario de larga duración la continuación del tratamiento médico o el cuidado del menor en domicilio tras el diagnóstico y hospitalización. La acreditación de que el menor padece un cáncer u otra

\footnotetext{
${ }^{17}$ BOE 23.12.2010.

${ }^{18}$ FUndación MuJer, TRABAJO y FAMILIA, Estudio sobre el cuidado de menores con enfermedad grave: una propuesta normativa, FIPROS, Instituto Seguridad Social, Madrid, 2011, p. 7.

${ }^{19}$ BOE 30.7.2011.
} 
enfermedad grave de las incluidas en el listado contenido en el anexo del texto reglamentario se efectuará mediante declaración cumplimentada por el facultativo del Servicio Público de Salud u órgano administrativo sanitario de la Comunidad Autónoma correspondiente, responsable de la atención del menor, incluso en aquellos casos en que la atención y diagnóstico del cáncer o enfermedad grave se haya llevado a cabo por servicios médicos privados -art. 2.2 RD 1148/2011-.

Serán beneficiarias del subsidio las personas trabajadoras, por cuenta ajena y por cuenta propia y asimiladas cualquiera que sea su sexo, que reduzcan su jornada de trabajo en, al menos, un 50\% de su duración, siempre que reúnan la condición general de estar afiliadas y en alta en algún régimen del sistema de la Seguridad Social y acrediten los periodos mínimos de cotización exigibles en cada caso. Para devengar esta prestación, el trabajador deberá reunir el período de carencia previsto para la prestación contributiva de cuidado por nacimiento, de modo si el trabajador tiene de 16 a 21 años de edad, se precisa un período de cotización de 90 días en los siete años anteriores al nacimiento de la prestación o, alternativamente, una carencia de 180 días durante toda su vida laboral; si es mayor de 26 años de edad, precisa una carencia de 180 días en los siete años anteriores o alternativamente 360 días a lo largo de su vida laboral; finalmente, si el trabajador es menor de 21 años no precisa carencia alguna.

El subsidio se reconocerá en proporción al porcentaje de reducción que experimente la jornada de trabajo en relación a la de una persona trabajadora a tiempo completo comparable de la misma empresa y centro de trabajo que realice un trabajo idéntico o similar, y se computará sin tener en cuenta otras reducciones de jornada que, en su caso, disfruten las personas trabajadoras por razones de guarda legal de menores o de cuidado de familiares, o por cualquier otra causa. Si en la empresa no hubiera ningún trabajador comparable a tiempo completo, se considerará la jornada prevista en el convenio colectivo de aplicación o, en su defecto, la jornada máxima legal.

Con su reconocimiento, el ordenamiento tutela un interés jurídicamente digno de protección: el cuidado de un menor enfermo a través de la creación de un subsidio que se incorpora al régimen público de Seguridad Social y que, por su propia configuración normativa, es compatible con el trabajo, por cuenta ajena o propia. A pesar de la valoración positiva que merece este desarrollo reglamentario, el mismo no resuelve si el derecho a disponer de la reducción de jornada puede calcularse en cómputo anual y no $\operatorname{diario}^{20}$.

Teniendo en cuenta que su finalidad es el acompañamiento de un menor durante el tiempo de hospitalización (distinto, por tanto, al interés tutelado en la reducción de jornada por guarda legal) y que, muchas veces, esta circunstancia conlleva el desplazamiento a centros sanitarios fuera de su comunidad de residencia, convendremos en la necesidad de

\footnotetext{
${ }^{20}$ Esta prestación es deudora del trabajo interdisciplinar que culminó con la publicación FuNDACIÓN Mujer, TrABAJO Y FAMILIA, Estudio sobre el cuidado de menores con enfermedad grave: una propuesta normativa, cit., p. 51.
} 
contemplar que esta reducción puede ser calculada en cómputo anual, incluso aunque no haya una previsión expresa en el convenio colectivo.

El riesgo subyacente de no admitirse esta interpretación finalista es que el progenitor encargado del cuidado del menor enfermo acuda a otras vías del sistema (v. gr. prestaciones de incapacidad temporal por contingencias comunes) para poder dar cobertura a esta situación de necesidad o sin el recurso al sistema, a través de una excedencia por cuidado de hijos con el menoscabo económico asociado. Evidentemente, el legislador podría haber alcanzado este resultado estableciendo dos posibilidades para articular esta prestación: de un lado, la reducción de jornada; de otro, mediante el reconocimiento de una suspensión contractual cuando un informe médico certificase la necesidad de que el cuidado sea continuo y que este alcance la totalidad de la jornada. Esta era, de otra parte, la propuesta planteada en el informe FIPROS ${ }^{21}$ en el que la citada prestación trae causa; en él expresamente se señalaba como "conveniente el disfrute a tiempo completo, como suspensión del contrato de trabajo en familias monoparentales, enfermedad o incapacidad de uno de los progenitores que por la reducción de sus capacidades físicas o psíquicas que se encuentre además de que la atención sólo pueda prestarse por otro progenitor o tutor".

Por todo lo es expuesto, es evidente que hubiese sido más conveniente que no se exigiese acuerdo previo con el empresario para la concreción horaria de la reducción, habida cuenta que se trata de una cuestión en la que debe primar la urgencia de la atención al menor que exclusivamente debieran valorar el facultativo y sus padres ${ }^{22}$. De no prosperar la tesis aquí propuesta, las posibles controversias en relación al ejercicio de este derecho tendrán que resolverse a través del procedimiento contenido en el art. 139 LRJS y en él, el juez tendrá que efectuar la correcta ponderación de las circunstancias concurrentes, valorando que en un supuesto como el expuesto también está en juego la salud de un menor.

La dimensión constitucional de todas aquellas medidas tendentes a facilitar la compatibilidad de la vida laboral y familiar de los trabajadores, tanto desde la perspectiva del derecho a la no discriminación por razón de sexo (artículo $14 \mathrm{CE}$ ) como desde la del mandato de protección a la familia y a la infancia (artículo $39 \mathrm{CE}$ ) ha de prevalecer y servir de orientación para la solución de cualquier duda interpretativa si existieran discrepancias para poder reducir la jornada en cómputo anual ${ }^{23}$, máxime en un supuesto tan específico como es el que da origen a esta prestación.

\footnotetext{
${ }^{21}$ Ibid., p. 31.

${ }^{22}$ Idéntica conclusión en Ibid., p. 50.

${ }^{23}$ García Testal, E.; LóPez BAlaguer, M., Los Derechos de conciliación de la vida laboral y familiar en la empresa, Tirant lo Blanch; Abdón Pedrajas, Valencia, 2012, p. 132.
} 


\section{La tímida apuesta por la corresponsabilidad en las últimas reformas normativas}

A pesar de que la Ley $39 / 1999^{24}$ pretendía o, al menos, este era el compromiso explicitado en su Exposición de Motivos "un nuevo modo de cooperación y compromiso entre mujeres y hombres que permita un reparto equilibrado de responsabilidades de la vida laboral y familiar", tempranamente se advirtió que la misma no estaba construida sobre la idea de corresponsabilidad sino en la individualización de los derechos de conciliación, manteniéndose la titularidad femenina en el permiso de lactancia y sin proceder a la regulación de un permiso de paternidad no transferible lo que perpetuaba la feminización de los cuidados. Hubo que esperar a la Ley Orgánica 3/2007 para que se acometiera la "plasmación legal de la corresponsabilidad de las labores domésticas y de atención a la familia".

Este avance se materializa en las referencias legales al término progenitor al tiempo que se "insiste en la necesidad de que los derechos de conciliación fomenten una mayor corresponsabilidad entre mujeres y hombres en la asunción de las obligaciones familiares y que su ejercicio no implique ningún tipo de discriminación para trabajadores y trabajadoras" 25 . Con esta regulación, basada en el reconocimiento de derechos autónomos, personales e intransferibles, se pretendía superar un modelo social caracterizado por repartir "las responsabilidades familiares en términos desproporcionados e injustos entre los hombres y las mujeres. Por consiguiente, cabe sostener que, precisamente, el objetivo último del derecho antidiscriminatorio en este campo se dirige a constatar y combatir dichas normas sociales. Es decir, al margen de las políticas concretas que se establezcan sobre la mejor conciliación de la vida familiar y laboral y sobre la corresponsabilidad de los varones, la prohibición de discriminación indirecta también se alza en contestación a un reparto equilibrado de la labor de cuidado" 26 .

Ahora bien, una mayor corresponsabilidad de hombres y mujeres en la tarea de cuidados no solo exige una reforma normativa sino un cambio radical en una norma social, no escrita pero vigente, que asume que los "cuidados" son responsabilidad de la mujer. En España, una amplia mayoría de la ciudadanía prefiere "una familia en la que los dos miembros de la pareja tienen un trabajo remunerado con parecida dedicación y ambos se reparten las tareas del hogar y el cuidado de los hijos/as, si los hay" (Barómetro del CIS, septiembre de 2010), pero como estos cuidados son muy intensivos en tiempo, es imprescindible que la sociedad asuma como norma una cogestión igualitaria de los cuidados entre hombres y mujeres desde el inicio, y esto implica que los permisos por maternidad/paternidad sean solitarios, intransferibles y, en la medida de lo posible, de igual duración.

\footnotetext{
${ }^{24}$ Ley 39/1999, de 5 de noviembre, para promover la conciliación de la vida familiar y laboral de las personas trabajadoras (BOE 6.11.1999).

${ }^{25}$ RodríGuez GonZÁlez, S., Tiempo de trabajo y vida privada, Comares, Granada, 2016, p. 19.

${ }^{26}$ BALlester PAstor, M. A.; J. CABeza Pereiro, J., "Retos, expectativas y decepciones del derecho a la conciliación de responsabilidades", cit., p. 109.
} 
Que el padre participe en solitario de los cuidados no solo permite "desfeminizarlos" sino que se avanza hacia la idea de cogestión de los mismos. La senda iniciada a este respecto por el ordenamiento español evidencia muy claramente la importancia de los permisos intransferibles, pues, aunque los derechos contenidos en los artículos 37.5 y 46.3 ET se configuran como derechos individuales de los trabajadores, hombres y mujeres, los datos de uso evidencian la feminización de estas instituciones.

A este respecto, el importante avance que supuso el reconocimiento de la suspensión del contrato por paternidad se hizo mediante su individualización, habida cuenta que el mismo "no puede transferirse bajo ninguna circunstancia y que, por lo tanto, se perderá si solo uno de los dos progenitores toma el permiso parental" 27 . En esta dirección, el RD Ley 6/2019, de 1 de marzo, de medidas urgentes para garantía de la igualdad de trato y de oportunidades entre mujeres y hombres en el empleo y la ocupación ${ }^{28}$, concretamente sus artículos 2 y 3 equipararon, en sus respectivos ámbitos de aplicación, la duración de los permisos por nacimiento de hijo o hija de ambos progenitores.

Esta equiparación responde, según el preámbulo del citado RD Ley, “a la existencia de una clara voluntad y demanda social. Los poderes públicos no pueden desatender esta demanda que, por otro lado, es una exigencia derivada de los artículos 9.2 y 14 de la Constitución; de los artículos 2 y 3.2 del Tratado de la Unión Europea; y de los artículos 21 y 23 de la Carta de los Derechos Fundamentales de la Unión Europea. De esta forma se da un paso importante en la consecución de la igualdad real y efectiva entre hombres y mujeres, en la promoción de la conciliación de la vida personal y familiar, y en el principio de corresponsabilidad entre ambos progenitores, elementos ambos esenciales para el cumplimiento del principio de igualdad de trato y de oportunidades entre hombres y mujeres en todos los ámbitos. Esta equiparación se lleva a cabo de forma progresiva, en los términos previstos en las disposiciones transitorias del Estatuto de los Trabajadores y del Estatuto Básico del Empleado Público”.

La existencia de permisos intransferibles ( $\mathrm{y}$, a ser posible, de igual duración, como ocurre en el estado español) es un factor determinante no solo para superar los estereotipos tradicionales de género -y el modelo tradicional de división sexual del trabajo- sino también para seguir avanzando hacia una mayor corresponsabilidad en el cuidado de menores y otras personas dependientes.

Al tiempo, es imprescindible reforzar la accesibilidad y la calidad de los servicios públicos de atención a menores y familiares enfermos o dependientes con el fin de que las personas con deberes de cuidado, significadamente mujeres, no se alejen de manera absoluta del mercado de trabajo ${ }^{29}$. Ya en el Consejo Europeo de Barcelona, celebrado los días 15 y 16 de marzo de 2002, se señalaba que "en cuanto a las actuales políticas de

\footnotetext{
${ }^{27}$ LÓPEZ TERRADA, E., “Permisos parentales y conciliación”, en Ballester Pastor, A. (ed.) La transposición del principio antidiscriminatorio comunitario al ordenamiento jurídico laboral español, Tirant lo Blanch, Valencia, 2010, p. 149.

${ }^{28}$ BOE 8.3.2021

29 RoDRíGUEZ GonZÁlez, S., "Desigualdad por causa de género en la Seguridad Social: carreras de cotización y prestaciones”, Lan harremanak: Revista de relaciones laborales, vol. 38, 2017, p. 123.
} 
empleo, los Estados miembros deberán suprimir los elementos que desincentivan la participación de la mano de obra femenina y, teniendo en cuenta la demanda de servicios de cuidado de niños y en consonancia con los modelos nacionales de asistencia, esforzarse en prestar para 2010 servicios de cuidado de niños al menos al 90 por ciento de los niños de edad comprendida entre los tres años y la edad de escolarización obligatoria, y al menos al 33 por ciento de los niños de menos de tres años".

Esta misma exigencia se reitera en el Pilar Social, tanto en el principio número 9 como en el 18 de los que parece deducirse una preocupación real de la UE por fomentar el acceso a servicios de cuidado, lo cual parece especialmente relevante, pues en aquellos Estados Miembros donde rige una amplia red de estas características, los beneficios advertidos son variados ${ }^{30}$, singularmente en relación al mantenimiento de las mujeres con responsabilidades familiares en el mercado de trabajo.

Lamentablemente, España está a la cola de los países de la Europa Occidental en cuanto al gasto público social que financia estos servicios; y, dentro de estos, "los menos financiados son los servicios de ayuda a las familias, incluyendo las escuelas de infancia desde los 0 años y los servicios de atención a las personas dependientes, tanto a domicilio como residenciales. El actual grado de (sub)desarrollo de todos estos servicios -servicios de dependencia, y servicios de educación a la primera infancia- se ha traducido en una enorme sobrecarga para la mujer, especialmente durante la pandemia, siendo su dedicación a las "responsabilidades familiares" (cuidado de sus niños y niñas y de las personas en situación de dependencia) el doble de la dedicación pre-pandemia"31.

La segunda decisión de política legislativa que trata de incrementar la corresponsabilidad de los hombres es el plan "MECUIDA", regulado en el art 6.1 RD Ley 8/2020, y prorrogado hasta el 31 de mayo de 2021, toda vez que contempla unos derechos de conciliación extraordinarios, partiendo de un "reparto corresponsable de las obligaciones de cuidado y la evitación de la perpetuación de roles".

Esta regulación supone la materialización en la ordenación jurídica de la previsión contenida la Ley Orgánica de Igualdad que señalaba a este respecto que "los derechos de conciliación de la vida personal, familiar y laboral se reconocerán a los trabajadores y las trabajadoras en forma que fomenten la asunción equilibrada de las responsabilidades familiares, evitando toda discriminación basada en su ejercicio" (LO 3/2007 art. 44.1).

El hecho de que las medidas se hayan hecho bascular sobre un reparto corresponsable de las obligaciones de cuidado y la evitación de la perpetuación de roles se materializa en la propia exclusión prevista por el legislador sobre los familiares afines dentro de los sujetos

\footnotetext{
${ }^{30}$ CRUZ ViLlalón, J., "Elementos condicionantes para la efectividad de la conciliación laboral en España", en Mella Méndez, L. (ed.) Conciliación de la vida laboral y familiar y crisis económica: estudios desde el derecho internacional y comparado, Delta Publicaciones Universitarias, Madrid, 2015, p. 25.

31 Véase, a este respecto, las reflexiones de Vicenç Navarro, "El abandono de las familias: el escaso desarrollo del cuarto pilar del bienestar", en https://blogs.publico.es/vicenc-navarro/2021/04/06/elabandono-de-las-familias-el-escaso-desarrollo-del-cuarto-pilar-del-bienestar/
} 
causantes $^{32}$. Exclusión que, además, resulta coherente con las previsiones de la Directiva $1158 / 2019$, supone "un avance hacia la deseada corresponsabilidad en las labores de cuidado y debe entenderse que el solicitante tiene que explicar cómo se repartirá para atender a esas tareas junto con el otro progenitor o cuidador que pudiese existir" ${ }^{33}$.

Ahora bien, aun admitiendo que el "presupuesto de las medidas adoptadas lo constituye el reparto corresponsable de las obligaciones de cuidado y la evitación de la perpetuación de roles" 34 , la empresa no podrá fiscalizar con respecto a la persona trabajadora solicitante el reparto corresponsable de las obligaciones de cuidado (con su pareja o con otros familiares potencialmente destinatarios de las obligaciones de cuidado) ni mucho menos evitar la perpetuación de roles.

El derecho ejercitado es un derecho de carácter individual y personalísimo, por lo que la persona solicitante no tiene por qué acreditar "la imposibilidad de acudir a otros mecanismos para conciliar la vida familiar y laboral, sin que tampoco tenga que probar que el marido u otra persona no pueda hacerse cargo de la menor" 35 .

Con todo, se debe aplaudir esta regulación ya que la misma "contribuye a la corresponsabilidad masculina en el cuidado de los familiares propios, por consanguinidad, y al reparto equitativo de las cargas familiares"36. En esta línea promocional, el Ministerio de Igualdad ha impulsado el "Plan Corresponsables", dotado con 190 millones de euros y que será ejecutado a través de los órganos competentes en materia de igualdad de las Comunidades Autónomas.

Este plan sigue la senda de algunas acciones autonómicas; a este respecto cabe significar el Plan de Corresponsabilidad de la Comunidad de Madrid, que se apoyará también en los ayuntamientos para promocionar un nuevo modelo de cuidados. Para ello, se fomentará la contratación de cuidadores, a través de las entidades locales, que prestarán sus servicios a todas aquellas familias que lo precisen, al tiempo que se contemplan otros incentivos como los destinados a la contratación de personas dadas de alta en el Sistema Especial de Empleadas del Hogar.

\footnotetext{
${ }^{32}$ RodríGuez Escanciano, S., "Medidas para la conciliación de la vida laboral y familiar ante la emergencia sanitaria por Covid-19", en Casas Baamonde, M. E., Rodríguez-Piñero, M. (eds.) Derecho del Trabajo y de la Seguridad Social ante la pandemia, Francis Lefebrvre, Madrid, 2020, p. 276.

${ }^{33}$ RodríGUez RodríGuez, E., "De la conciliación a la corresponsabilidad en el tiempo de trabajo: un cambio de paradigma imprescindible para conseguir el trabajo decente", cit., p. 56.

${ }^{34}$ BARRIOS BAUDOR, G., "Adaptaciones y/o reducciones especiales de jornada con ocasión de la crisis sanitaria COVID-19: Plan MECUIDA", cit.

${ }^{35}$ STSJ Galicia 6.11.2020 (R' 2046/2020)

${ }^{36}$ RodríGuez Escanciano, S., "Medidas para la conciliación de la vida laboral y familiar ante la emergencia sanitaria por Covid-19”, en Casas Baamonde, M. E., Rodríguez-Piñero, M. (eds.) Derecho del Trabajo y de la Seguridad Social ante la pandemia, Francis Lefebrvre, Madrid, 2020, p. 276.
} 


\section{Las medidas para mitigar la brecha prestacional. El insuficiente alcance de las acciones impulsadas}

El sistema de cuidado referido en los epígrafes precedentes, con sus innegables connotaciones de género, se proyecta de manera inmediata en el Derecho de la Seguridad Social, cuyas técnicas de protección basculan sobre referentes temporales varios (edades de jubilación, acreditación de carencias, períodos de cálculo de las bases reguladoras...) que suelen perjudicar a las mujeres, toda vez que cuando ellas acceden masivamente al mercado de trabajo se encuentran con un "sistema cimentado en una distribución lineal del tiempo difícilmente compatible con las imposiciones de género, de ahí que, o las mujeres las eluden, lo que casi nunca resulta fácil (y, si ello supusiera una renuncia a la maternidad, resultaría simplemente contrario a sus derechos), o las asumen a costa de realizar una doble jornada que, para desarrollarla dignamente, les empuja con cierta frecuencia al trabajo a tiempo parcial"37.

Las peores carreras de seguro repercuten tanto en el número de beneficiarias como en la cuantía de las pensiones lucradas por mujeres ${ }^{38}$, toda vez que el tiempo cotizado al sistema es, por término medio, menor en el caso de las mujeres que de los hombres, pero es que, además, al presentar carreras de cotización más cortas, las mujeres han cotizado al sistema por bases de menor cuantía.

Esta realidad provoca, en el ámbito de la protección social, dos consecuencias claras: a) Una mayor dificultad para acceder a las prestaciones de carácter contributivo: la edad media de jubilación de las mujeres (64,3 años) es superior a la de los hombres (63,5 años); y b) una menor cuantía de las pensiones lo que favorece una brecha prestacional ${ }^{39}$ que, en lo que se refiere a la jubilación, es palmaria: los pensionistas obtienen un importe medio de 1.303,82 frente a los 848,44 euros de las pensionistas ${ }^{40}$, siendo más mujeres que hombres los que tienen reconocido un complemento por mínimos (correctivo legal que se aplica a la cuantía de la pensión cuando no alcance el mínimo fijado legalmente) en su pensión de jubilación. Pero es que, además, cuando termine el plazo de la regulación transitoria contenida en la Ley 27/2011, de 1 de agosto, sobre actualización, adecuación y modernización del sistema de Seguridad Social ${ }^{41}$, para el cálculo de la jubilación se van a computar las bases de los 300 meses anteriores al hecho causante, es decir, 25 años de carrera profesional, y parece bastante factible, con los datos ya referidos, que las mujeres

\footnotetext{
${ }^{37}$ Martínez Barroso, M. R., "Discriminación en el acceso a la pensión de jubilación contributiva de las trabajadoras a tiempo parcial: a propósito de la STJUE de 22 de noviembre de 2012 (Asunto Elbal Moreno)", Revista General de Derecho Europeo, vol. 31, 2013, p. 4.

${ }^{38}$ Benito Benítez, M. A., El impacto de género en el sistema de pensiones, Bomarzo, Albacete, p. 28.

39 Véanse, a este respecto, las atinadas reflexiones de la profesora Aragón Gómez en: https://forodelabos.blogspot.com/2019/07/un-nuevo-avance-en-la-reduccion-de-la.html $\quad$ (Fecha de consulta: 9/2/2021).

40 Sobre los mismos, puede consultarse: https://www.bbva.com/es/brecha-genero-pensiones-diferenciapensiones-percibidas-hombres-mujeres-alcanza-34/

${ }^{41}$ BOE 2.8.2011.
} 
no puedan aportar este período de cotización de forma continua, sin lagunas ${ }^{42}$, especialmente por su concentración en el trabajo a tiempo parcial ${ }^{43}$.

Para tratar de mitigar esta situación, la DA $5^{\text {a }}$ de la Ley 27/2011 encargó al Gobierno que elaborase un estudio con propuestas de actuación en relación con la Recomendación $17^{\mathrm{a}}$ del Pacto de Toledo que, como es de sobra conocido, ya abogaba por la aprobación de medidas en el ámbito de la Seguridad Social que permitiesen a las mujeres generar derechos propios, concretamente reclamaba el impulso de mecanismos que incorporasen los periodos de atención y cuidado de los hijos o personas dependientes como elementos a considerar en las carreras de cotización ${ }^{44}$.

Este mandato fue parcialmente atendido en el Texto Refundido de la LGSS, aprobado por RD Legislativo 8/2015, de 30 octubre, pues, dentro del Capítulo XV del Título II, rubricado "Protección a la familia" y estructurado en 3 artículos (arts. 235-237), sistematiza una serie de medidas, cuyo denominador común es que con su activación se asimila a tiempo cotizado determinados periodos dedicados al cuidado de hijos o familiares dependientes ${ }^{45}$. Esta decisión, coherente con las líneas de actuación aprobadas en el Pacto Europeo por la Igualdad de Género (2011-2020) y con la ya referida recomendación del Pacto de Toledo, relativa a "Mujer y protección social", parte de la necesidad de valorar la dimensión de género en materia de pensiones.

Aunque las medidas correctoras ponen en valor una función social tan importante como es el cuidado e implican una materialización de la obligación constitucional de asegurar la protección de la familia por los poderes públicos ${ }^{46}$, su configuración legal es claramente insuficiente ${ }^{47}$. La mejor acción en relación con la brecha prestacional es la eliminación de la brecha salarial, pues la primera no deja de ser el anverso de la segunda. Para lograr esta reducción es necesaria una decidida apuesta por la corresponsabilidad en los

\footnotetext{
${ }^{42}$ Las mujeres ya tienen más dificultad para acceder a la jubilación parcial, toda vez que una vez acreditados los requisitos generales y específicos, el importe de la pensión a percibir ha de resultar superior a la cuantía de la pensión mínima que correspondería al interesado por su situación familiar al cumplimiento de los 65 años. En caso contrario, no se podrá acceder a esta fórmula de jubilación anticipada. La mejor doctrina ya aboga por introducir un correctivo que facilite a las mujeres el acceso a la jubilación anticipada, ARAGón GÓMEZ, C., "El impacto de la conciliación en el acceso a la protección del sistema de Seguridad Social", en AA.VV. (ed.) Protección a la familia y Seguridad Social. Hacia un nuevo modelo de protección social, Laborum, Murcia, 2018, p. 137.

${ }^{43}$ BLÁZQUeZ AGUdo, E., "Trabajo de las mujeres y protección social", en AEDTSS (ed.) El futuro del trabajo: cien años de la OIT. XXIX Congreso Anual de la Asociación Española de Derecho del Trabajo y la Seguridad Social, Ministerio de Trabajo, Migraciones y Seguridad Social, Madrid, 2019, p. 182.

${ }^{44}$ García Romero, B., "Medidas de Seguridad Social en respuesta a los retos que plantean las responsabilidades familiares: fomento de la natalidad, conciliación e igualdad de género prestacional", Revista de derecho social, vol. 74, 2016, p. 123.

${ }^{45}$ ARAGÓN GÓMEZ, C., "El impacto de la conciliación en el acceso a la protección del sistema de Seguridad Social", cit., pp. 128-133.

${ }^{46}$ Blázquez Agudo, E.; Barrios Baudor, G., Prestaciones familiares, Aranzadi, Navarra, 2009, p. 174.

47 Véanse las atinadas críticas de la profesora Sánchez Rodas al complemento de maternidad en: https://revistascientificas.us.es/index.php/erips/article/view/13150 (Fecha de consulta: 11/3/2021).
} 
cuidados, pues como bien señalaba la Proposición de Ley de Tiempo de Trabajo Corresponsable $^{48}$, presentada por el Grupo Parlamentario Unidas-Podemos:

"Los hábitos de distribución y organización del tiempo propio, aunque han experimentado una evolución significativa, siguen evidenciando una tendencia a una distribución de los tiempos lineal en los hombres, alternando trabajo y tiempo libre, y en estrella en las mujeres, superponiendo tiempos de trabajo productivo, doméstico y ocio. En el caso de las mujeres, su desproporcionada dedicación al cuidado de la familia ha sostenido un sistema de protección informal en España que termina descargando en ellas las responsabilidades de cuidado que debería compartir toda la sociedad". La repercusión del este cuidado en su situación profesional se encuentra sobradamente constatada puesto que las mayores diferencias retributivas entre hombres y mujeres se producen en los años en los que normalmente se desarrolla la crianza; de hecho, tales efectos perversos se causan no solo si dicha crianza se produce efectivamente, sino incluso ante la mera posibilidad de que pudiera producirse.

Como hemos analizado, la peor situación de la mujer en el mercado de trabajo "tiene un claro impacto en su carrera de seguro a efectos de causar, en el futuro, una prestación de la Seguridad Social. En efecto, los datos evidencian que el tiempo cotizado al sistema es, por término medio, más alto en el caso de los hombres que en el de las mujeres y esta diferencia, además, se va incrementando con la edad.

Mientras que los hombres entre 45 y 65 años han estado cotizando por término medio un 90,4\% del tiempo total transcurrido desde su entrada en el sistema de la Seguridad Social, en el caso de las mujeres esta proporción sólo llega al 82,6\% ${ }^{49}$. A mayor abundamiento, durante los períodos de no empleo, los hombres tienen más episodios en los que están cobrando la prestación por desempleo que las mujeres y ya sabemos que, durante el paro, la entidad gestora asume las cotizaciones del beneficiario"50.

No es exagerado afirmar que la brecha de género constituye la principal insuficiencia en la acción protectora de la Seguridad Social en el ámbito de las pensiones, y la misma no es más que "un reflejo de una discriminación histórica y estructural de las mujeres en el mercado de trabajo por la asunción del rol de cuidadoras de los hijos e hijas" ${ }^{\text {. }}$.

La maternidad afecta decisivamente a la trayectoria laboral de la mujer en su etapa en activo y es esta una, si no la más importante, causa de esa brecha: cuanto mayor es el número de hijos, menor es el número de años cotizados, menor es la proporción de contratos a tiempo completo o equivalente, y menor es, en última instancia, la pensión reconocida. Urgen, por tanto, medidas legales que traten "de reparar un perjuicio que han

\footnotetext{
${ }^{48}$ BOCG 18.11.2018.

${ }^{49}$ CeBrián LóPeZ, I.; MoReno RAYMundo, G., "Tiempo cotizado, ingresos salariales y sus consecuencias para las pensiones: diferencias por género al final de la vida laboral”. Cuadernos de Relaciones Laborales, 2015, vol. 33, núm 2, p. 320.

${ }^{50}$ ARAGÓN GómEZ, C., "El impacto de la conciliación en el acceso a la protección del sistema de Seguridad Social”, cit., p. 121.

${ }^{51}$ Preámbulo RD Ley 3/2021, de 2 de febrero, por el que se adoptan medidas para la reducción de la brecha de género y otras materias en los ámbitos de la Seguridad Social y económico (BOE 3.2.2021).
} 
sufrido a lo largo de su carrera profesional las mujeres que hoy acceden a la pensión, es decir, un perjuicio generado en el pasado, y que, por tanto, resulta perfectamente compatible y coherente con el desarrollo de políticas de igualdad ambiciosas que corrijan las desigualdades actualmente existentes en el mercado de trabajo y la asignación de los roles relacionados con los cuidados" 52 , y, en este contexto, tiene que hacerse una breve mención al complemento a las pensiones contributivas para la reducción de la brecha de género, regulado en el art. 60 LGSS, aunque su análisis será objeto de atención específica en este monográfico.

El RD Ley 3/2021 prevé que "las mujeres que hayan tenido uno o más hijos o hijas y que sean beneficiarias de una pensión contributiva de jubilación, de incapacidad permanente o de viudedad, tendrán derecho a un complemento por cada hijo o hija, debido a la incidencia que, con carácter general, tiene la brecha de género en el importe de las pensiones contributivas de la Seguridad Social de las mujeres. El derecho al complemento por cada hijo o hija se reconocerá o mantendrá a la mujer siempre que no medie solicitud y reconocimiento del complemento en favor del otro progenitor y si este otro es también mujer, se reconocerá a aquella que perciba pensiones públicas cuya suma sea de menor cuantía. Prevé la nueva regulación que para que los hombres puedan tener derecho al reconocimiento del complemento deberá concurrir alguno de los siguientes requisitos: a) Causar una pensión de viudedad por fallecimiento del otro progenitor por los hijos o hijas en común, siempre que alguno de ellos tenga derecho a percibir una pensión de orfandad; b) Causar una pensión contributiva de jubilación o incapacidad permanente y haber interrumpido o haber visto afectada su carrera profesional con ocasión del nacimiento o adopción" en los términos referidos en la LGSS.

El nuevo art. 60 LGSS, aunque se construye a partir de una medida de acción positiva se adecua a las exigencias del TJUE al permitir "una "vía abierta" para aquellos varones $u$ otro progenitor que puedan acreditar el perjuicio en su carrera de cotización con ocasión del nacimiento o adopción de una hija/o, con la expresa previsión de que "sin ninguno de los progenitores acreditara perjuicio en la cotización, el "complemento, lo percibe la mujer" ${ }^{\text {" }}$, enmendando a través de esta vía la regulación anterior. Regulación que fue calificada por la Sentencia del Tribunal de Justicia de la Unión Europea, de 12 de diciembre de 2019, (asunto WA) ${ }^{54}$, como contraria "a la Directiva 79/7/CEE del Consejo, de 19 de diciembre de 1978, relativa a la aplicación progresiva del principio de igualdad de trato entre hombres y mujeres en materia de seguridad social". Entendió el TJUE que resultaba discriminatorio que se reconociera un derecho a un complemento de pensión por aportación demográfica para las mujeres (con, al menos, dos hijos), "mientras que los

\footnotetext{
${ }^{52}$ Reflexión contenida en el preámbulo del Real Decreto-ley 3/2021, de 2 de febrero, por el que se adoptan medidas para la reducción de la brecha de género y otras materias en los ámbitos de la Seguridad Social y económico (BOE 3.2.2021).

${ }^{53}$ Valorando este RD Ley, las atinadas reflexiones de Romero Ródenas en el siguiente enlace: https://baylos.blogspot.com/search?updated-max=2021-02-07T10:49:00\%2B01:00\&maxresults $=3 \&$ start $=6 \&$ by - date $=$ false

${ }^{54}$ El texto puede consultarse en: http://curia.europa.eu/juris/liste.jsf?num=C-450/18\&amp;language=ES (Fecha de consulta: 3 de marzo de 2021).
} 
hombres que se encuentran en una situación idéntica no tienen derecho a tal complemento". La norma española suponía un trato menos favorable a los hombres con, al menos, dos hijos biológicos o adoptados, lo cual constituía, a juicio del alto tribunal europeo, una discriminación directa por razón de sexo, prohibida por la Directiva, al entender que "dado que la aportación de los hombres a la demografía es tan necesaria como la de las mujeres, la aportación demográfica a la Seguridad Social no puede justificar por sí sola que los hombres y las mujeres no se encuentren en una situación comparable en lo que respecta a la concesión del complemento de pensión controvertido" $" 55$.

\section{A modo de conclusión}

Los datos analizados en este artículo evidencian que las mujeres siguen asumiendo la mayor parte de las responsabilidades familiares y domésticas, y que este cuidado tiene un notable impacto en sus trayectorias laborales; varias son las causas de este diferente reparto de responsabilidades:

El principal motivo parece la tradicional división de tareas entre los sexos, que atribuye al varón el papel de sostenedor económico de la familia y a la mujer la responsabilidad de atender el hogar y a los miembros que lo integran. El segundo motivo, podría deberse al miedo a la señalización: los hombres que hacen uso de los permisos parentales, en la medida en que forman parte de un colectivo minoritario, podrían enviar a la empresa más señales de falta de compromiso con su entorno laboral que las propias mujeres. De hecho, resulta especialmente elocuente que el porcentaje de hombres que ha solicitado la baja por paternidad sea superior en el sector público $(89 \%)$ que en el sector privado $(70 \%)^{56}$. El tercer motivo, no despreciable, es fruto de la brecha salarial entre hombres y mujeres, pues, salvo la adaptación de jornada o las suspensiones contractuales que generan, si se acredita el período de carencia necesario, una prestación del sistema de la Seguridad Social, las restantes medidas de conciliación de la vida familiar y profesional conllevan una merma de los ingresos familiares, lo que favorece el hecho de una pareja encuentre mayores incentivos a prescindir del salario más bajo (normalmente el de la mujer) ${ }^{57}$, teniendo un correlato en su brecha prestacional.

Urge también un nuevo diseño de los servicios a las personas dependientes, estableciendo un sistema público de atención a la dependencia, con servicios suficientes y de calidad y condiciones laborales dignas para las personas que trabajan y trabajarán en este sector, lo cual debería también ocurrir en los servicios de atención y educación a la primera

55 Sobre el impacto de la STJUE, puede verse la nota de prensa en: https://curia.europa.eu/jcms/upload/docs/application/pdf/2019-12/cp190154es.pdf

${ }^{56}$ MEIL LANDWERLIN, G. Y ROMERO BALSAS, P. «Los permisos parentales para el cuidado de niños: percepción social y usos de los mismos entre padres y madres». En: Tamayo Haya, Silvia. La maternidad y paternidad en el S. XXI. Granada: Comares, 2016, p. 247, que advierten que las condiciones laborales más estables del sector público podrían estar detrás de estos resultados.

${ }^{57}$ Aragón Gómez, C.; Nieto Rojas, P., Planes de igualdad. Procedimiento de creación e implementación, Wolters Kluwer, Madrid, 2021, p. 174. 
infancia, que también deberían ser públicos, suficientes y de calidad ${ }^{58}$. La pertinencia de avanzar en la gestión pública de los cuidados tiene dos efectos añadidos: en primer lugar, porque, ante un Estado que actúa de manera insuficiente, las familias, o más correctamente, las mujeres asumen la responsabilidad de este cuidado, teniendo, por ello, peores trayectorias laborales que los hombres. De conformidad con la EPA, las mujeres realizan, de media, una jornada semanal inferior en cinco horas a la jornada semanal de los hombres ${ }^{59}$, y de esta misma encuesta se deprende que, del $100 \%$ de ocupados a tiempo parcial un 74,37\% son mujeres ${ }^{60}$. En segundo lugar, porque muchas de las carencias de los servicios públicos de atención a la dependencia acaban siendo suplidas mediante la contratación de una mujer ajena al ámbito familiar, en el precarizado sector del empleo doméstico, y, con esta decisión, "los problemas de la crisis de los cuidados se transfieren de unas mujeres a otras, en base a ejes de poder, en una larga cadena de la cual están sistemáticamente ausentes los hombres -o, en todo caso-, presentes únicamente en los eslabones finales" $"$.

\section{Bibliografía}

Almendros GonZÁlez, M A., "La reforma de la coordinación de las prestaciones familiares de seguridad social como respuesta a los cambios políticos y económicos en la Unión Europea", Revista General de Derecho del Trabajo y de la Seguridad Social, vol. 49, 2018 , p. 5.

ÁlVAREZ RAMíreZ, G., Estudio sobre las necesidades de las familias con personas con discapacidad, Ministerio de Sanidad, Servicios Sociales e Igualdad, Madrid, 2015.

ARAGÓN GÓMEZ, C., "El impacto de la conciliación en el acceso a la protección del sistema de Seguridad Social", en AA.VV. (ed.) Protección a la familia y Seguridad Social. Hacia un nuevo modelo de protección social, Laborum, Murcia, 2018.

Aragón Gómez, C.; Nieto Rojas, P., Planes de igualdad. Procedimiento de creación e implementación, Wolters Kluwer, Madrid, 2021.

Ballester Pastor, M. A.; Cabeza Pereiro, J., J., "Retos, expectativas y decepciones del derecho a la conciliación de responsabilidades", en Cruz Villalón, J. (ed.) Tutela y promoción de la plena integración de la mujer en el trabajo: libro homenaje a la

\footnotetext{
${ }^{58}$ A este respecto en

${ }^{59}$ INE. Encuesta de Población Activa. Ocupados. Número medio de horas efectivas semanales trabajadas por los ocupados que han trabajado por situación profesional, sexo y rama de actividad (empleo principal). Datos correspondientes al 4T 2020.

${ }^{60}$ INE. Encuesta de Población Activa. Ocupados. Ocupados a tiempo parcial por motivo de la jornada parcial, sexo y grupo de edad. Datos correspondientes al 4T 2020.

${ }^{61}$ PÉrez OrozCO, A. Amenaza tormenta: la crisis de los cuidados y la reorganización del sistema feminista, Revista de Economía Crítica, no 5. Marzo de 2006, p. 26.
} 
profesora Teresa Pérez del Río, Consejo Andaluz de Relaciones Laborales, Sevilla, 2015, pp. 103-138.

BARRIOS BAUDOR, G., "Adaptaciones y/o reducciones especiales de jornada con ocasión de la crisis sanitaria COVID-19: Plan MECUIDA", Revista Aranzadi Doctrinal, vol. 6, 2020 .

Benito Benítez, M. A., El impacto de género en el sistema de pensiones, Bomarzo, Albacete.

BLÁZQuez Agudo, E., "Trabajo de las mujeres y protección social”, en AEDTSS (ed.) El futuro del trabajo: cien años de la OIT. XXIX Congreso Anual de la Asociación Española de Derecho del Trabajo y la Seguridad Social, Ministerio de Trabajo, Migraciones y Seguridad Social, Madrid, 2019.

Blázquez Agudo, E.; Barrios Baudor, G., Prestaciones familiares, Aranzadi, Navarra, 2009.

CAstro ArgüElles, M. A., "La protección familiar en el sistema español de Seguridad Social: un enfoque general”, en González Ortega, S. (ed.) Seguridad Social y Familia, La Ley, Madrid, 1999.

CRUZ Villalón, J., "Elementos condicionantes para la efectividad de la conciliación laboral en España”, en Mella Méndez, L. (ed.) Conciliación de la vida laboral y familiar y crisis económica: estudios desde el derecho internacional y comparado, Delta Publicaciones Universitarias, Madrid, 2015, pp. 5-26.

García Romero, B., "Medidas de Seguridad Social en respuesta a los retos que plantean las responsabilidades familiares: fomento de la natalidad, conciliación e igualdad de género prestacional”, Revista de derecho social, vol. 74, 2016, pp. 113-138.

García Testal, E.; LóPez Balaguer, M., Los Derechos de conciliación de la vida laboral y familiar en la empresa, Tirant lo Blanch; Abdón Pedrajas, Valencia, 2012.

Grau PinedA, C., La brecha de las pensiones en España, Bomarzo, Albacete, 2021.

LÓPEZ TerradA, E., "Permisos parentales y conciliación”, en Ballester Pastor, A. (ed.) La transposición del principio antidiscriminatorio comunitario al ordenamiento jurídico laboral español, Tirant lo Blanch, Valencia, 2010, pp. 17-75.

Martínez Barroso, M. R., "Discriminación en el acceso a la pensión de jubilación contributiva de las trabajadoras a tiempo parcial: a propósito de la STJUE de 22 de noviembre de 2012 (Asunto Elbal Moreno)", Revista General de Derecho Europeo, vol. 31, 2013, p. 12.

Molinier, P., “Género, trabajo y sexualidad”, en Laufer, L., Rochefort, F. (eds.) ¿Qué es el género?, Icaria, Barcelona, 2016, 
MuÑoz Molina, J., "La mujer en el ordenamiento de Seguridad Social”, en Sáez Lara, C. (ed.) Igualdad de género en el trabajo: estrategias y propuestas, Laborum, Murcia, 2016.

PÉREz Orozco, A. Amenaza tormenta: la crisis de los cuidados y la reorganización del sistema feminista, Revista de Economía Crítica, nº 5. Marzo de 2006.

RODRÍGUEZ COPE, M. L., "Igualdad, conciliación, corresponsabilidad y flexibilidad", en Sáez Lara, C. (ed.) Igualdad de género en el trabajo: estrategias y propuestas, Laborum, Murcia, 2016.

RODRÍGUEZ EsCANCIANO, S., "Medidas para la conciliación de la vida laboral y familiar ante la emergencia sanitaria por Covid-19”, en Casas Baamonde, M. E., RodríguezPiñero, M. (eds.) Derecho del Trabajo y de la Seguridad Social ante la pandemia, Francis Lefebrvre, Madrid, 2020.

RoDRíGUEZ GonZÁLEZ, S., "Desigualdad por causa de género en la Seguridad Social: carreras de cotización y prestaciones", Lan harremanak: Revista de relaciones laborales, vol. 38, 2017, pp. 93-125.

Rodríguez GonZÁlez, S., Tiempo de trabajo y vida privada, Comares, Granada, 2016.

\section{Anexo}

CONSEJO ECONÓMICO Y SOCIAL, El cuidado de la primera infancia en los hogares españoles.

Fundación Mujer, Trabajo y FAMiLia, Estudio sobre el cuidado de menores con enfermedad grave: una propuesta normativa, FIPROS, Instituto Seguridad Social, Madrid, 2011.

ILO, Care work and care jobs for the future of decent work, ILO, Geneva, 2018. 